\title{
The General Expression of the Diurnal Variation of the Atmospheric Electric Field Considering the Influence of the Eddy Diffusion near the Ground.
}

\author{
By Minoru KAWANO \\ Electrotechnical Laboratory, Tokyo, Japan \\ (Read Oct. 11, 1957 ; Received .Nov. 10, 1957)
}

\begin{abstract}
The diurnal variation curves of the atmospheric electric field, conductivity and space charge at Hongo, Tokyo, were described, and the local anomaly of these curves in urban condition was fairly well clarified.

Considering the influence of eddy diffusion, we discussed the ionization equilibrium process near the ground. The result of the calculation permitted us to estimate the vertical distribution of the air resistivity. Using the vertical distribution, the local characteristic of the atmospheric electric field was derived on the theoretical basis from the fundamental equation.

According to the results, the atmospheric electric field is proportional to the space charge and the square root of the coefficient of eddy diffusivity. This consideration shows that the diurnal variation of the atmospheric electric field can be explained by the fundamental equation of the electro-magnetic field assuming the Ohm's law.
\end{abstract}

\section{Introduction}

The several authors [1], [2], [3] have made comparisons of diurnal variations of the atmospheric electric field observed at many stations on land in the world. Mühleisen [4] has carried out the simultaneous observations at two stations, which are situated in comparatively short distance in the different condition each other, and concluded that the complicated variation in the urban condition seems to be caused by the positive space charge which is originated densely by the industrial activities in the urban condition. Kawano [5] has clarified that we must take into consideration the influence of the convection current near the ground to explain the stationary change in short duration.

But, the comparison of the diurnal variations of the electric field, conductivity and space charge at two stations situated in the different conditions each other was not carried out.

In this paper, the diurnal variation curves of these three elements obtained at Hongo, Tokyo, situated in urban condition are described, and compared them with those obtained by Aoki and others [6], [7], [8] at Tanashi, situated at $25 \mathrm{~km}$ apart from Hongo.

Considering that the stationary change in each element of the atmospheric 
electricity is controlled by the ionization equilibrium process, we will discuss the local anomaly of the diurnal variation of the atmospheric electric field of the basis of the fundamental equation.

\section{The diurnal variation of the atmospheric electric field, conductivity and space charge at Hongo}

A few examples of the record of the atmospheric electric field are shown in Fig. 1. The mean hourly values of the electric field in each month are shown in July $14 \sim 15,1950$

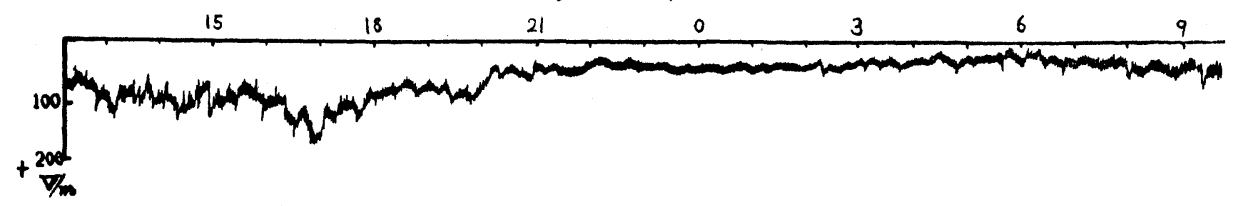

Dec. $19 \sim 20,1950$

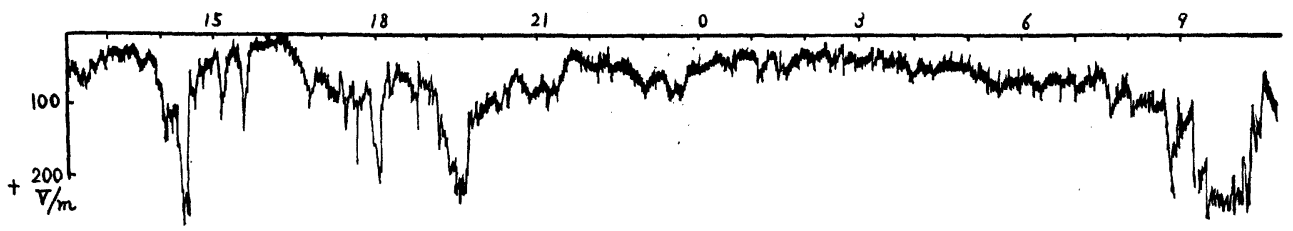

Fig. 1 The examples of th observed record of the atmospheric electric field at Hongo.

Table 1. In Fig. 2-4 are shown the mean diurnal variation curves of the electric field, space charge and conductivity in summer and winter. Uses are made of the record covering the period from Aug. 1949 to Feb. 1952. The seasonal change can be readily seen in the figures. Another interesting thing is that in winter, the conductivity is in general low and the electric field is high, but as the year progresses into the summer the conductivity increases while the electric field decreases. The most interesting feature is the remarkable difference between diurnal variation in summer and winter. In winter season the variation is generally regular in character, and the maximum value of the electric field occurs at about $10 \mathrm{~h}$ L.M.T.. In summer season the diurnal

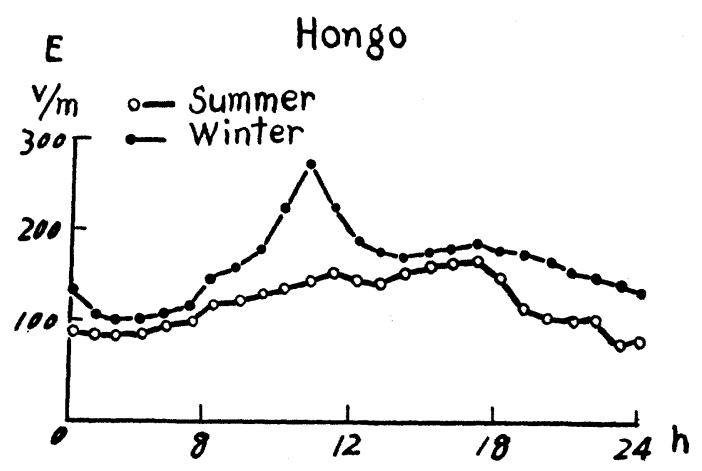

Fig. 2 The diurnal variation curves of the electric field at Hongo.

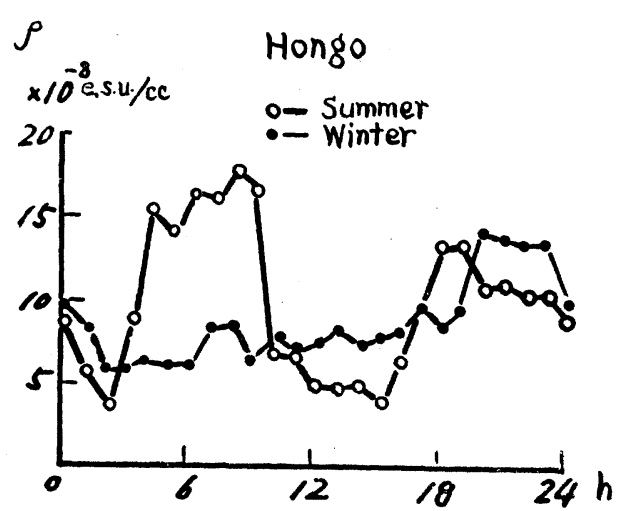

Fig. 3 The diurnal variation curves of the space charge at Hongo. 


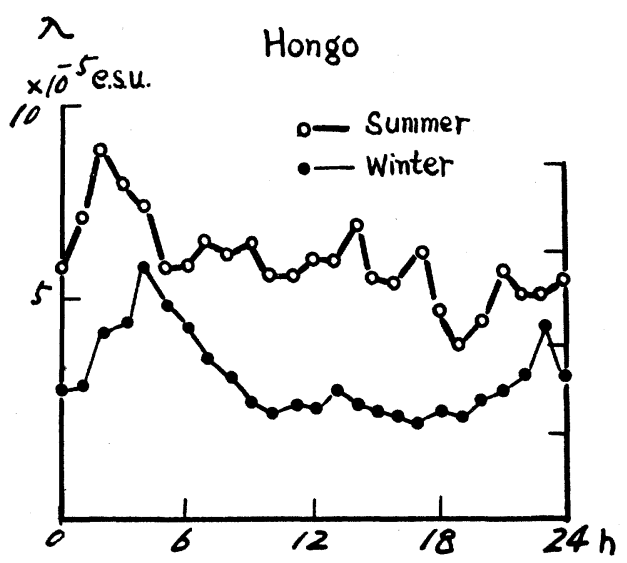

Fig. 4 The diurnal variation curves of the conductivity at Hongo. variation curve is broken and irregular having the maximum at about 17 L.M.T.. The conductivity curves show less seasonal difference. They are almost regular throughout the year, but the times of maximum and minimum change somewhat occuring at $02 \mathrm{~h}$ during the hot season and at about $03 \mathrm{~h}$ during the cold season respectively.

The diurnal variation of space charge seems to be not correlated with that of the electric field and conductivity. But, the diurnal variation curve of space charge has its own characteristic, and its maximum value occures at about $8 \mathrm{~h}$ L.M.T. throughout the year.

Table 1 Atmospheric electric field (unit: $\mathrm{V} / \mathrm{m}$ )

\begin{tabular}{|c|c|c|c|c|c|c|c|c|c|c|c|c|}
\hline $\begin{array}{r}\text { time } \\
\text { month }\end{array}$ & 1 & 2 & 3 & 4 & 5 & 6 & 7 & 8 & 9 & 10 & 11 & 12 \\
\hline Jan. & 99 & 100 & 100 & 115 & 120 & 135 & 142 & 210 & 270 & 255 & 220 & 200 \\
\hline Feb. & 106 & 106 & 107 & 112 & 102 & 140 & 146 & 162 & 230 & 270 & 230 & 190 \\
\hline Mar. & 85 & 775 & 70 & 90 & 125 & 139 & 140 & 190 & 225 & 260 & 235 & 195 \\
\hline Apr. & 70 & 55 & 60 & 60 & 85 & 100 & 130 & 170 & 200 & 220 & 190 & 170 \\
\hline May & 55 & 50 & 50 & 60 & 80 & 100 & 120 & 150 & 175 & 210 & 200 & 185 \\
\hline June & 41 & 55 & 100 & 137 & 128 & 167 & 159 & 200 & 186 & 194 & 239 & 155 \\
\hline July & 133 & 139 & 120 & 110 & 113 & 129 & 150 & 130 & 143 & 129 & 122 & 191 \\
\hline Aug. & 84 & 76 & 52 & 53 & 60 & 70 & 98 & 90 & 113 & 124 & 118 & 134 \\
\hline Sept. & 40 & 30 & 41 & 47 & 67 & 79 & 79 & 131 & 123 & 136 & 132 & 132 \\
\hline Oct. & 71 & 77 & 70 & 73 & 77 & 89 & 103 & 120 & 156 & 234 & 210 & 210 \\
\hline Nov. & 143 & 133 & 114 & 120 & 156 & 148 & 194 & 200 & 220 & 230 & 260 & 228 \\
\hline Dec. & 110 & 100 & 90 & 115 & 120 & 175 & 180 & 220 & 250 & 300 & 270 & 240 \\
\hline
\end{tabular}

\begin{tabular}{rrrrrrrrrrrr}
\hline 13 & 14 & 15 & 16 & 17 & 18 & 19 & 20 & 21 & 22 & 23 & 24 \\
\hline 170 & 150 & 150 & 170 & 170 & 175 & 180 & 170 & 160 & 150 & 140 & 130 \\
170 & 164 & 184 & 204 & 199 & 174 & 190 & 183 & 172 & 169 & 157 & 145 \\
100 & 170 & 165 & 155 & 170 & 190 & 190 & 160 & 140 & 130 & 100 & 95 \\
140 & 125 & 135 & 130 & 120 & 120 & 130 & 110 & 105 & 90 & 75 & 80 \\
130 & 125 & 120 & 120 & 130 & 140 & 145 & 120 & 115 & 80 & 80 & 65 \\
144 & 126 & 159 & 169 & 167 & 198 & 140 & 126 & 119 & 114 & 92 & 84 \\
170 & 180 & 162 & 194 & 197 & 180 & 118 & 106 & 109 & 117 & 101 & 118 \\
134 & 207 & 190 & 177 & 176 & 160 & 102 & 99 & 92 & 92 & 75 & 67 \\
138 & 120 & 106 & 114 & 110 & 103 & 95 & 63 & 76 & 70 & 90 & 44 \\
178 & 180 & 159 & 176 & 170 & 167 & 135 & 130 & 73 & 87 & 65 & 80 \\
211 & 180 & 215 & 220 & 245 & 260 & 230 & 198 & 210 & 173 & 160 & 176 \\
200 & 190 & 170 & 175 & 180 & 190 & 170 & 160 & 145 & 140 & 135 & 130 \\
\hline
\end{tabular}


The partial conductivity observed at Hongo was the only positive one. So long as the electric field is less than about $300 \mathrm{~V} / \mathrm{m}$, the electrode effect of the atmospheric electric field seems not to be so effective. Accordingly, it may be permissible to consider that the total electric conductivity is proportional to the positive partial conductivity in the stationary state. Comparing the diurnal vai iations of the three elements obtained in various seasons of the year, there exist considerable differences among them both in magnitude and characters, and that of the electric field is specially outstanding in the grade of difference. The most remarkable difference between Hongo and Tanashi [6], [7], [8] is the seasonal change of the mode of diurnal variation curves of the electric field. The maximum value of electric field occurs at $17 \mathrm{~h}$ in summer and $10 \mathrm{~h}$ in winter at Hongo, and $8 \mathrm{~h}$ in both seasons at Tanashi. As the diurnal variation curves of the electric field at Tanashi coincide fairly well with those at other stations situated in rural condition, the seasonal change seems to be the most remarkable characteristic at Hongo situated in urban condition.

\section{Fundamental equations}

The atmospheric electric field in the stationary state may be described by the equations.

$$
\begin{aligned}
& \nabla \times \boldsymbol{H}=\boldsymbol{i} \\
& \nabla \times \boldsymbol{E}=0 \\
& \boldsymbol{\nabla} \cdot \boldsymbol{B}=0 \\
& \nabla \cdot \boldsymbol{D}=\boldsymbol{\rho}
\end{aligned}
$$

Since the conduction current by the large ion is negligible small, the conduction current in the atmospheric electric field seems to depend only upon the small ion. So, we may assume the following relation:

$$
\boldsymbol{i}=\lambda \boldsymbol{E}
$$

Further, we shall assume that

$$
\boldsymbol{D}=\varepsilon \boldsymbol{E}
$$

Putting (6) into (4), we get

$$
\nabla \cdot(\varepsilon \boldsymbol{E})=\rho
$$

It follows from (2) that

$$
\boldsymbol{\nabla} \cdot \boldsymbol{i}=0
$$

Then, from (5) and (8), we have

$$
\boldsymbol{\nabla} \cdot(\lambda \boldsymbol{E})=0
$$

Referring to (7), we get

$$
\boldsymbol{\nabla} \cdot(\varepsilon \boldsymbol{E})=\boldsymbol{\nabla} \cdot\left(\frac{\varepsilon}{\lambda}-\lambda \boldsymbol{E}\right)=\lambda \boldsymbol{E} \cdot \boldsymbol{\nabla}\left(\frac{\varepsilon}{\lambda}\right)+\frac{\varepsilon}{\lambda} \boldsymbol{\nabla} \cdot(\lambda \boldsymbol{E})=\rho
$$

Substituting (5) into (10), we get

From (11), we get

$$
\boldsymbol{E} \cdot \nabla\left(\begin{array}{l}
\varepsilon \\
\lambda
\end{array}\right)=\frac{\rho}{\lambda}
$$

$$
\boldsymbol{E} \cdot \nabla(\log \lambda)=-\frac{\rho}{\varepsilon}
$$


From (2), we get

$$
\boldsymbol{E}=-\nabla \phi
$$

Putting (13) into (9), we have

$$
\nabla \cdot(\lambda \nabla \phi)=0
$$

Assuming that both of $\lambda$ and $\phi$ are functions of the radial component of the spherical coordinate, we get from equation (14):

$$
\frac{1}{r^{2}} \frac{d}{d r}\left(r^{2} \lambda \frac{d}{d r} \phi\right)=0
$$

Integrating (15), we get

$$
\begin{aligned}
& \frac{d}{d r} \phi=\frac{C}{\lambda(r) r^{2}} \\
& \boldsymbol{E}=\boldsymbol{n}_{r} E_{r} ; \boldsymbol{n}_{r}: \text { unit vector } \\
& E_{r}=-\frac{d}{d r} \phi=-\frac{C}{\lambda(r) r^{2}}
\end{aligned}
$$

Total current $I$ can be given by the following relation:

$$
I=\int_{0}^{2 \pi} d \varphi \int_{0}^{\pi}\left(\boldsymbol{i} \cdot \boldsymbol{n}_{r}\right) r^{2} \sin \theta d \theta
$$

Here,

$$
\boldsymbol{i}=\lambda \boldsymbol{E}=-\boldsymbol{n}_{r} \frac{C}{r^{2}}
$$

From (18) and (19), we get

$$
I=\int_{0}^{2 \pi} d \varphi \int_{0}^{\pi} \lambda E_{r} r^{2} \sin \theta d \theta=-4 \pi C \quad(20), \quad \therefore \quad C=-\frac{I}{4 \pi}
$$

Putting (21) into (17) and (19), we get

$$
\boldsymbol{i}=\boldsymbol{n}_{r} \frac{I}{4 \pi r^{2}}, \quad \boldsymbol{E}=\boldsymbol{n}_{r} \frac{I}{4 \pi \lambda r^{2}}
$$

Substituting (22) into (7), we get as follows.

$$
\rho=\varepsilon \frac{1}{r^{2}} \frac{d}{d r}\left(r^{2} \frac{I}{4 \pi r^{2} \lambda}\right)=\frac{\varepsilon I}{4 \pi r^{2}} \frac{d}{d r}\left(\frac{1}{\lambda}\right)
$$

Here, $4 \pi r^{2}$ is the total surface area of the earth. Accordingly, $I /\left(4 \pi r^{2}\right)$ is equal to the conduction current density at the earth's surface and will be expressed by the symbol $i$ hereafter. Considering the small area of the earth's surface, and assuming $\varepsilon$ being 1 in the air, we can write the formula (23) as follows:

$$
\rho=i \frac{d}{d z}\left(\frac{1}{\lambda}\right)
$$

Where $z$ is the vertical axis of the orthogonal coordinate. Using the electrostatic unit, we can write (24) as follows.

$$
\rho=\frac{i}{4 \pi} \frac{d}{d z}\left(\begin{array}{l}
1 \\
\lambda
\end{array}\right)
$$

The formula $\left(24^{\prime}\right)$ gives the relation among the space charge, conduction current density and the vertical distribution of the air-resistivity. 


\section{The vertical distribution of the electrical conductivity of the atmosphere near the ground}

Taking into account the influence of the eddy diffusion, we may describe the process of the ionization equilibrium by the following equation.

$$
K \frac{d^{2} n}{d z^{2}}+q=\alpha n^{2}+\beta N n
$$

Here, $K, \alpha$ and $\beta$ are the coefficient of eddy diffusivity, coefficient of recombination of the small ion and the coefficient of attachment between the small ion and nuclei respectively. And, $q, n$ and $N$ are the rate of ion pair production, small ion number and nuclei content respectively. The left hand of equation (25) expresses the sum of the net flux of ions due to the eddy diffusion and the rate of ion pair production by the all kind of ionization in the reference volume, and the right hand expresses the sum of the rate of ion destruction by recombination and attachment in the same volume.

Over land, especially near centres of pollution, the values of $N$ are often observed so large that the first term of right hand side of (25) can be ignored compared to the second. In these cases, the equation (25) may be written as follows.

$$
K \frac{d^{2} n}{d z^{2}}+q=\beta N n
$$

The ionizing agents of the atmosphere near the ground are as follows:

i) Radiation by the radioactive gases and their decay products in the atmosphere.

ii) Penetrating radiation by the radioactive subtances in the earth's crust.

iii) Cosmic ray.

Considering that the quantities of radiation of cosmic ray and penetrating radiation from the earth's crust are not affected by air motions, we may say that the rate of ion pair production by these two ionizing agents are independent of air motions. On the other hand, the distribution of the radioactive gases and their decay products in the atmosphere are affected directly by air motions as discussed in the previous paper (9). Therefore the rate of ion pair production by the radiation from these substances is influenced by air motions. Then, we may classify these three ionizing agents into two groups :

i) Radiation by the radioactive gases and their decay products in the atmosphere, which is affected by air motions.

ii) Penetrating radiations by the radioactive substances in the earth's crust and cosmic ray, which are not affected by air motions.

Let us denote by $q_{1}$ and $q_{2}$ the rates of ion pair production by the ionizing agents i) and ii) respectively. Then, we may write as follows:

$$
q=q_{1}+q_{2}
$$

Assuming that the vertical distribution of the radioactive substance in the atmosphere is controlled by the eddy diffusivity, the distribution of the substance is expressed by the following formula. Here, $\tau$ and $K$ are the decay constant of $\mathrm{Rn}$ and the coefficient of eddy diffusivity respectively. 


$$
Q=Q_{h} e^{-\sqrt{7 K \cdot(z-h)}}
$$

The ionization due to the radioactive substances in the atmosphere is mainly caused by $\alpha$ rays. Consequently, the rate of ion pair production, due to the radiation by the radioactive substances in the atmosphere, may be considered to be proportional to the content of the radioactive substances in the atmosphere.

In other word, we may write:

$$
q_{1}=k_{1} Q
$$

where $k_{1}$ is a constant.

Substituting (28) into (27), we get the following formula.

$$
q_{1}=q_{1 h} e^{-\sqrt{\tau \cdot K} \cdot(z-h)}
$$

where $q_{1, i}$ is the value of the rate of ion pair production at $z=h$.

Then, $q$ is given by

$$
q=q_{1 / h} e^{-\sqrt{7 / K} \cdot(2-h)}+q_{2}
$$

Referring to $\left(25^{\prime}\right)$, the equation form becomes

$$
K \frac{d^{2} n}{d z^{2}}+q_{1 h} e^{-\sqrt{7 / K} \cdot(z-h)}+q_{2}=\beta N n
$$

Boundary conditions for $n$ are

$$
\begin{array}{ll}
n=n_{h} & \text { at } z=h \\
n: \text { finite } & \text { at } z=\infty
\end{array}
$$

The solution of equation (31) is found to be that:

$$
n=\left\{n_{h}-\left(\frac{q_{1 h}}{\beta N-\tau}+\frac{q_{2}}{\beta N}\right)\right\} e^{-\sqrt{\beta N / K} \cdot(z-h)}+\frac{q_{1 i}}{\beta N-\tau} e^{-\sqrt{\tau / K} \cdot(z-h)}+\frac{q_{2}}{\beta N}
$$

The vertical distribution of the electrical conductivity of the atmosphere near the ground is given by the following formula.

$$
\lambda=n e k=\left[\left\{n_{h}-\left(\frac{q_{1 h}}{\beta N-\tau}+\frac{q_{2}}{\beta N}\right)\right\} e^{-\sqrt{\beta N / K} \cdot(z-h)}+\frac{q_{1 h}}{\beta N-\tau} e^{-\sqrt{\tau / K} \cdot(z-h)}+\frac{q_{2}}{\beta N}\right] e k
$$

Where $k$ is the mobility of small ion, and assumed to be constant. Accordingly, the vertical distribution of the air resistivity near the ground is given by the following formula.

$$
r=1 /\left[\left\{n_{i}-\left(\frac{q_{1 h}}{\beta N-\tau}+\frac{q_{2}}{\beta N}\right)\right\} e^{-\sqrt{\beta N} \mid K \cdot(z-h)}+\frac{q_{1 h}}{\beta N-\tau} e^{-\sqrt{\tau / K} \cdot(z-h)}+\frac{q_{2}}{\beta N}\right] e k
$$

\section{The relation between the atmospheric electric field and the other elements near the ground}

Differentiating the formula (34), we get

$$
\frac{d r}{d z}=\frac{\left(\frac{\beta N}{K}\right)^{1 / 2}\left\{n_{i}-\left(\frac{q_{1 h}}{\beta N-\tau}+\frac{q_{2}}{\beta N}\right)\right\} e^{-\sqrt{\beta N / K} \cdot(z-h)}+\left(\frac{\tau}{K}\right)^{1 / 2} \frac{q_{1 h}}{\beta N-\tau} e^{-\sqrt{\tau / K} \cdot(z-h)}}{\left[\left\{n_{h}-\left(\frac{q_{1 i}}{\beta N-\tau}+\frac{q_{2}}{\beta N}\right)\right\} e^{-\sqrt{\beta N / K} \cdot(z-h)}+\frac{q_{1 h}}{\beta N-\tau} e^{-\sqrt{7 / K}(z-h)}+\frac{q_{2}}{\beta N}\right]^{2} e k}
$$


Substituting (35) into $\left(24^{\prime}\right)$, we get the following formula.

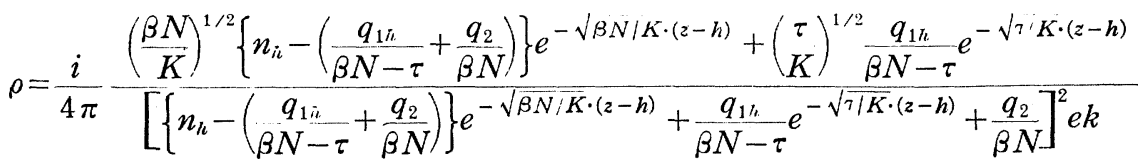

$$
\begin{aligned}
& =\frac{n_{h} E}{4 \pi} \frac{\left(\frac{\beta N}{K}\right)^{1 / 2}\left\{n_{h}-\left(\begin{array}{c}
q_{1 h} \\
\beta N-\tau
\end{array}+\frac{q_{2}}{\beta N}\right)\right\} e^{-\sqrt{\beta N / K} \cdot(z-h)}+\left(\frac{\tau}{K}\right)^{1 / 2} \frac{q_{1 h}}{\beta N-\tau} e^{-\sqrt{\tau / K} \cdot(z-h)}}{\left[\left\{n_{h}-\left(\frac{q_{1 h}}{\beta N-\tau}+\frac{q_{2}}{\beta N}\right)\right\} e^{-\sqrt{\beta N / K \cdot(z-h)}}+\frac{\left.q_{1 h}-e^{-\sqrt{\tau / K} \cdot(z-h)}+\frac{q_{2}}{\beta N}\right]^{2}}{\beta N-\tau}\right.}
\end{aligned}
$$

At height $h$, this relation becomes

$$
\begin{aligned}
\rho_{i t} & =\frac{n_{i} E_{i}}{4 \pi} \frac{\left(\frac{\beta N}{K}\right)^{1 / 2}\left\{n_{i}-\left(\frac{q_{1 i}}{\beta N-\tau}+\frac{q_{2}}{\beta N}\right)\right\}+\left(\begin{array}{c}
\tau \\
K
\end{array}\right)^{1 / 2} \begin{array}{c}
q_{1 h} \\
\beta N-\tau
\end{array}}{n_{h}{ }^{2}} \\
& =\frac{E_{i}}{4 \pi} \frac{\left(\frac{\beta N}{K}\right)^{1 / 2}\left\{n_{i}-\left(\frac{q_{1 h}}{\beta N-\tau}+\frac{q_{2}}{\beta N}\right)\right\}+\left(\begin{array}{c}
\tau \\
K
\end{array}\right)^{1 / 2} \frac{q_{1 h}}{\beta N-\tau}}{n_{h}}
\end{aligned}
$$

This formula gives the relation among the elements of the atmospheric electricity at height $h$ above the ground.

Considering that the value of $\tau$ is $2.09 \times 10^{-6} \mathrm{sec} .^{-1}$, and that of $\beta N$ is nearly $10^{-2}$ sec. ${ }^{-1}$ on land, (37) may be reduced to

$$
\rho_{i h}=\frac{E_{i}}{4 \pi} \frac{\left(\frac{\beta N}{K}\right)^{1 / 2}\left(n_{i t}-\frac{q_{1 h}+q_{2}^{-}}{\beta N}\right)}{n_{i}}
$$

From (38), we get

$$
E_{i t}=4 \pi \rho_{i i} \frac{n_{h} K^{1 / 2}}{(\beta N)^{1 / 2}\left(n_{i i}-\frac{q_{1 i}+q_{2}}{\beta N}\right)}
$$

The formula (39) expresses the electric field considering the influence of the eddy diffusion on the vertical distribution of the electrical conductivity of the atmosphere. The formula (39) is rewritten as

$$
N=\frac{1}{\beta}\left[\left(\frac{q_{1 i}+q_{2}}{n_{i}}\right)^{1 / 2}+\frac{2 \pi \rho_{i i}}{E_{h}} K^{1 / 2}+\frac{1}{2}\left(\begin{array}{c}
q_{1 h t}+q_{2} \\
n_{i h}
\end{array}\right)^{-1 / 2}\left(\begin{array}{c}
2 \pi \rho_{i t} \\
E_{i}
\end{array}\right)^{2} K\right]^{2}
$$

This formula gives the nuclei content near the ground. Here, the hourly values of $E_{i}, \rho_{i i}, n_{i i}$ are shown in Fig. 2 4. $q_{1 i}+q_{2}$ and $K$ are shown in the other paper (9) (10). The value of $\beta$ employed here, is $5 \times 10^{-6} \mathrm{~cm}^{3} \mathrm{sec}^{-1}$

Using these hourly values and (40), the diurnal variation of the nuclei content can be computed. The results are shown in Fig. 5 for summer and winter seasons. As we can see in this figure, the calculated values of the nuclei content are 8,000 $-14,000 \mathrm{cc}^{-1}$ in summer, and $12,000-22,000 \mathrm{cc}^{-1}$ in winter. The mode of diurnal variation curve in summer is similar to that in winter, and the number of $N$ is large at night and small in the daytime. Although the routin observation of $N$ was not carried out at Hongo, the values observed by Aitken counter at Nishigahara in summer, 1953 
were about $10,000 \mathrm{cc}^{-1}$ at night and about $8,000 \mathrm{cc}^{-1}$ in the daytime. We may say that the calculated values coincide fairly well with the observed values. Fig. 6 shows the diurnal variation curve of the dust concentration observed by Koshi (11) at Shiba, Tokyo. In this figure, $Y$ is used for the unit of the dust concentration, and it is almost proportional to the nuclei content. The modes of the computed diurnal variation curves and the seasonal change, shown in Fig. 5, coincide fairly well with observed ones. It appeares the good coincidence that the atmospheric electric field near the ground can be expressed considerably well by the formula (40). Morever, from the results noted above we may say the atmospheric electric field is proportional to the

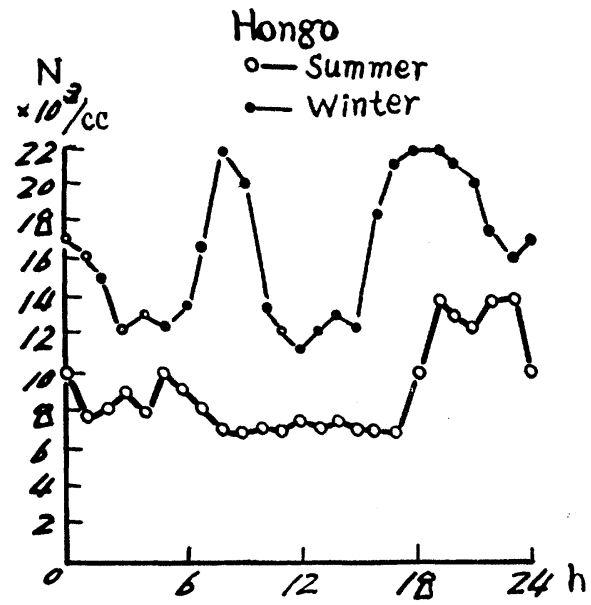

Fig. 5 The estimated diurnal variation curves of the nuclei content at Hongo.

Tanashi

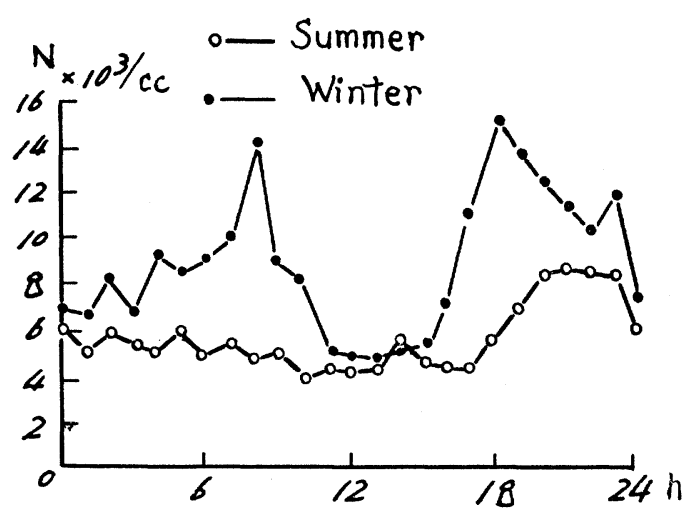

Fig. 7 The estimated diurnal viaration curves of nuclei content at Tanashi.

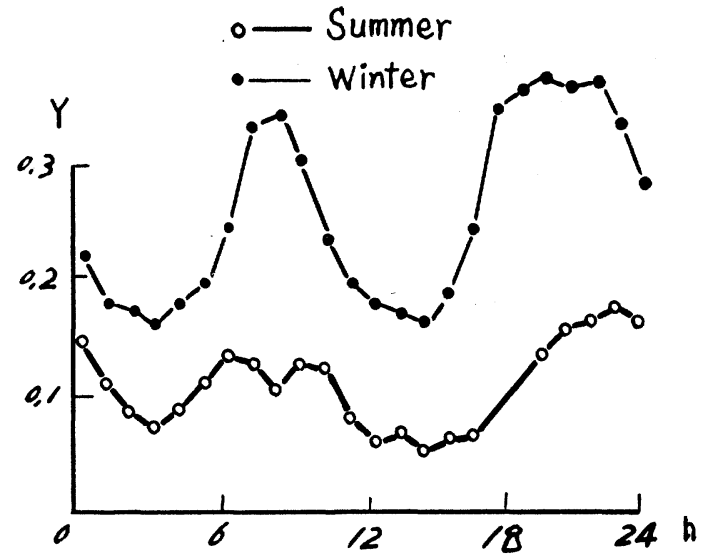

Fig. 6 The observed diurnal variation curves of nuclei content in Tokyo.

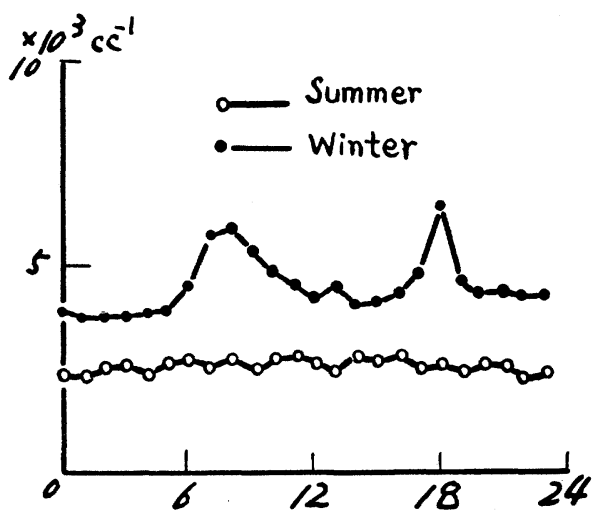

Fig. 8 The observed diurnal variation curves of large ion number at Tanashi.

product of the space charge and the square root of the coefficient of eddy diffusivity. Although the discussions mentioned above are concerned with the atmospheric electric field at Hongo, no generality is lost in the discussion. Consequently, the diurnal variation of the atmospheric electric field in the suburban or rural conditions may be explained 
in the same way. In Fig. 7, shown is the diurnal variation of the nuclei content, $N$, for Tanashi, computed from (40). Uses are made of the observed values of the atmospheric electric field, space charge and ion number at Tanashi. [6], [7], [8]

Since the rate of ion pair production by the penetrating radiation from the ground surface is not measured at Tanashi, the value obtained at Hongo is used. Fig. 8 shows the diurnal variation curve of the large ion number observed at Tanashi [12]. According to the results of observations the nuclei content is almost proportional to the large ion number. Consequently, the correspondence between the computed diurnal variation of the nuclei content and the diurnal variation of the large ion number observed in suburban condition may be again regarded as a guarantee for the presumption that the formula (40) give the considerably correct expression of the atmospheric electric field in the suburban condition.

From the consideration mentioned above, the formula (40) seems to explain the local characteristic of the atmospheric electric field on land.

\section{Conclusion}

Considering the influence of the eddy diffusion, we discussed the ionization equilibuim near the ground and obtained the vertical distribution of the electrical conductivity of the atmosphere. Using the vertical distribution thus derived, we can explain the local characteristic of the electric field on land on the basis of the fundamental equation. The formula of the electric field is expressed as follows:

$$
E_{h}=4 \pi \rho_{h} \frac{n_{h} K^{1 / 2}}{(\beta N)^{1 / 2}\left(n_{h}-\frac{q_{1 h}+q_{2}}{\beta N}\right)}
$$

\section{Acknowledgement}

The writer wishes to express his sincere thanks to Prof. T. Nagata, Tokyo University, for his permission of using the whole data obtained at Geophysical Institute, Tokyo University, and to his guidance and encouragement throughout this work.

\section{References}

[1] Mauchly, S.J.: Terr. Mag. Atm. Elec.. 28, 61 (1923).

[2] Brown, J.G.: Terr. Mag. Atm. Elec., 40, 413 (1935).

[3] Hatakeyama, H. and M. Kawano: Pap. Met. Geophys., 4, 55 (1935).

[4] Mühleisen, R.: Zeit. Geophys., 142 (1953).

[5] Kawano, M.: Journ. Geomag. Geoelec., 5, 16 (1953).

[6] Aoki, T.: Researches of the Electrotechnical Laboratory, No. 494 (1949).

[7] Aoki, T. and K. Kato: Journ. Electrotechnical Laboratory, 18, 562 (1954).

[8] Kawasaki, K. and K. Kato: Journ. Electrotechnical Laboratory, 16, 200 (1952).

[9] Kawano, M.: Journ. Met. Soc. Japan, 35, No. 6 (1957).

[10] Kawano, M.: Journ. Met. Soc. Japan, 35, No. 6 (1957).

[11] Koshi, S. : Journ. Met. Soc. Japan, 34, 327 (1956).

[12] Aoki, T. and K. Kato: Journ. Electrotechnical Laboratory, 17, 339 (1953). 